\title{
The scope of Analytical and Bioanalytical Chemistry (ABC)
}

\author{
H. Cui ${ }^{1}$ • P. Garrigues ${ }^{1}$ - G. Gauglitz ${ }^{1}$ E. Hilder ${ }^{1}$ - G. Hopfgartner ${ }^{1}$ • D. C. Muddiman ${ }^{1}$

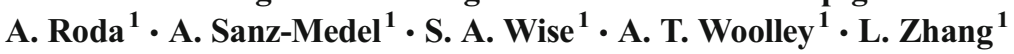

Published online: 22 November 2017

(C) Springer-Verlag GmbH Germany, part of Springer Nature 2017

One of the most common questions that we are asked by authors is whether their proposed article fits the scope of Analytical and Bioanalytical Chemistry (ABC). With a mission of rapid publication of excellent and high-impact research articles on fundamental and applied topics of analytical and bioanalytical measurement science, Analytical and Bioanalytical Chemistry has a necessarily broad scope that ranges from novel measurement platforms and their characterization to multidisciplinary approaches that effectively address important scientific decisions. A more detailed description is provided in our recently revised Aims and Scope: "The Editors encourage submissions presenting innovative analytical research in concept, instrumentation, methods, and/or applications, including mass spectrometry, spectroscopy, and electroanalysis; advanced separations; analytical strategies in "-omics" and imaging, bioanalysis, and sampling; miniaturized devices, medical diagnostics, and sensors; analytical characterization of nano- and biomaterials; and chemometrics and advanced data analysis."

We do recognize that such a list can never be exhaustive. In determining the suitability of manuscripts for publication in Analytical and Bioanalytical Chemistry, the most critical consideration remains how the work

Published in the topical collection celebrating ABCs 16th Anniversary.

1 Analytical and Bioanalytical Chemistry, Springer, Tiergartenstraße 17, 69121 Heidelberg, Germany demonstrates an innovative contribution to the field. With this in mind, what are the key factors used to determine this? In many cases, innovation can be shown through introduction of a new analytical or bioanalytical method or through significant improvement or advancement relative to an existing method. Whether introducing a new method or demonstrating an improvement on an existing method, novelty is best demonstrated through direct comparison with existing methods which can show in what way performance is improved. For the authors, a good question to aim to answer is "why would the reader choose to use our method over an existing method?" Regardless of the novelty, in determining whether a method is within the scope of Analytical and Bioanalytical Chemistry, a good guide is to see if we have recently published similar articles and if your manuscript cites work in this and/or related journals.

Analytical and Bioanalytical Chemistry also encourages submission of manuscripts on novel applications. This may include applying a known analytical or bioanalytical method to a new and/or challenging application, and should clearly demonstrate the analytical challenge solved and how this may impact on further research in the field. It may also involve analysis of rare or unusual samples addressing important scientific issues. In assessing whether an application is within the scope of Analytical and Bioanalytical Chemistry, we recommend that authors consider whether the major focus of the manuscript is to advance another field. Regardless of the quality of these manuscripts, if they do not include a new or improved analytical method, they may not fit within the scope of Analytical and Bioanalytical Chemistry. 
We hope that this editorial provides some further insight into the scope of Analytical and Bioanalytical Chemistry and we look forward to your submissions focused on new funda- mental developments and applications in analytical and bioanalytical measurement science.

The ABC Editors.

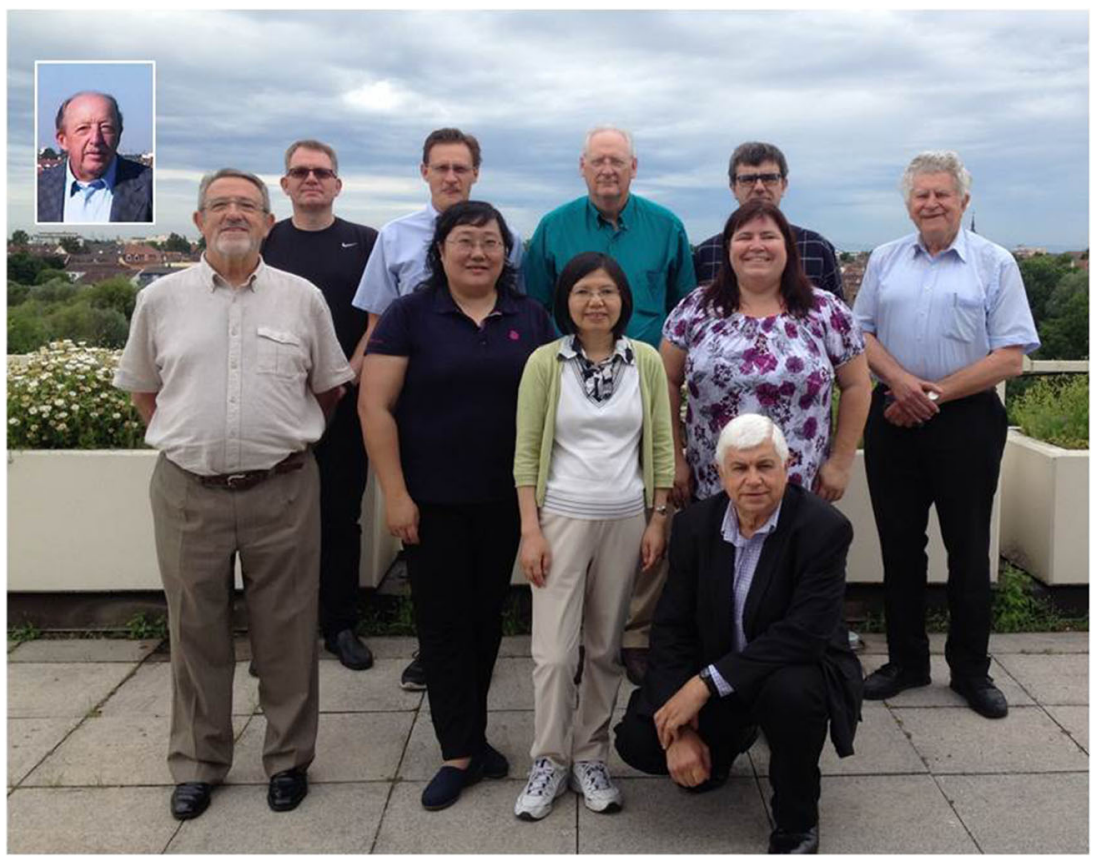

The ABC Editors at one of their annual meetings; from left to right: Alfredo Sanz-Medel, David Muddiman, Adam Woolley, Lihua Zhang, Hua Cui, Stephen Wise, Emily Hilder, Gérard Hopfgartner, Günter Gauglitz, and Philippe Garrigues in the front row; Aldo Roda was not able to attend in 2017. 